\title{
Nanotechnology in regenerative medicine: the materials side
}

\author{
Elisabeth Engel, Alexandra Michiardi, Melba Navarro, Damien Lacroix \\ and Josep A. Planell
}

Institute for Bioengineering of Catalonia (IBEC), Department of Materials Science, Technical University of Catalonia, CIBER BBN, Barcelona, Spain

\begin{abstract}
Regenerative medicine is an emerging multidisciplinary field that aims to restore, maintain or enhance tissues and hence organ functions. Regeneration of tissues can be achieved by the combination of living cells, which will provide biological functionality, and materials, which act as scaffolds to support cell proliferation. Mammalian cells behave in vivo in response to the biological signals they receive from the surrounding environment, which is structured by nanometre-scaled components. Therefore, materials used in repairing the human body have to reproduce the correct signals that guide the cells towards a desirable behaviour. Nanotechnology is not only an excellent tool to produce material structures that mimic the biological ones but also holds the promise of providing efficient delivery systems. The application of nanotechnology to regenerative medicine is a wide issue and this short review will only focus on aspects of nanotechnology relevant to biomaterials science. Specifically, the fabrication of materials, such as nanoparticles and scaffolds for tissue engineering, and the nanopatterning of surfaces aimed at eliciting specific biological responses from the host tissue will be addressed.
\end{abstract}

\section{Introduction}

Regenerative medicine has brought high expectations for a great number of current worldwide human illnesses. Diseases, such as Parkinson's disease, Alzheimer's disease, osteoporosis, spine injuries or cancer, might in the near future be treated with methods that aim at regenerating diseased or damaged tissues. The perspective of regenerating damaged or nonfunctional tissues by using an off-theshelf synthetic product is a drive for medical science. Today's interest in nanomedicine keeps growing because the application of nanotechnology tools to the development of structures at the molecular level enables the improvement of the interactions between material surfaces and biological entities. Although cells have micrometre dimensions, they evolve in vivo in close contact with the extracellular matrix (ECM), a substratum with topographical and structural features of nanometre size. The interactions between cells and the ECM influence cell growth, guide cell mobility and affect the general behaviour of cells. Nanotechnologies provide the possibility to produce surfaces,

Corresponding author: Planell, J.A. (Josep.A.Planell@upc.edu). structures and materials with nanoscale features that can mimic the natural environment of cells, to promote certain functions, such as cell adhesion, cell mobility and cell differentiation.

Nanomaterials used in biomedical applications include nanoparticles for molecules delivery (drugs, growth factors, DNA), nanofibres for tissue scaffolds, surface modifications of implantable materials or nanodevices, such as biosensors. The combination of these elements within tissue engineering (TE) is an excellent example of the great potential of nanotechnology applied to regenerative medicine. The ideal goal of regenerative medicine is the in vivo regeneration or, alternatively, the in vitro generation of a complex functional organ consisting of a scaffold made out of synthetic or natural materials that has been loaded with living cells (Figure 1). Ideally, stem cells are to be used owing to their ability to generate all types of tissues and their unlimited self-renewal capacity. The functionalisation of such a porous scaffold with different biomolecules (depending on the targeted cells) or the entrapment of nanoparticles, such as growth factors, drugs or genes, could enhance the success of the TE strategy greatly. However, crucial issues, such as stem cell isolation from the patient and their proliferation, the culturing process in a bioreactor and the time delay before the engineered hybrid construct is implanted back into the patient, present major difficulties to this approach becoming a well established standardised procedure in the near future.

The aim of this article is to review the aspects related to the materials used currently for tissue regeneration and it will furthermore point out the most promising strategies in the field. Other equally important issues, such as cell therapies or diagnostic applications, fall out of the scope of this review and will not be addressed.

\section{Nanofeatures on surface biomaterials}

As discussed earlier, the behaviour of cells depends on their interactions with the environment. Consequently, the interactions between cells and implantable materials will determine the success or failure of a medical device. It is well known that cell response is affected by the physicochemical parameters of the biomaterial surface, such as surface energy, surface charges or chemical composition. Topography is one of the most crucial physical cues for cells. Microtopography influences cell adhesion, proliferation and differentiation [1,2] and, more recently, it has become clear that nanotopography also guides cell 


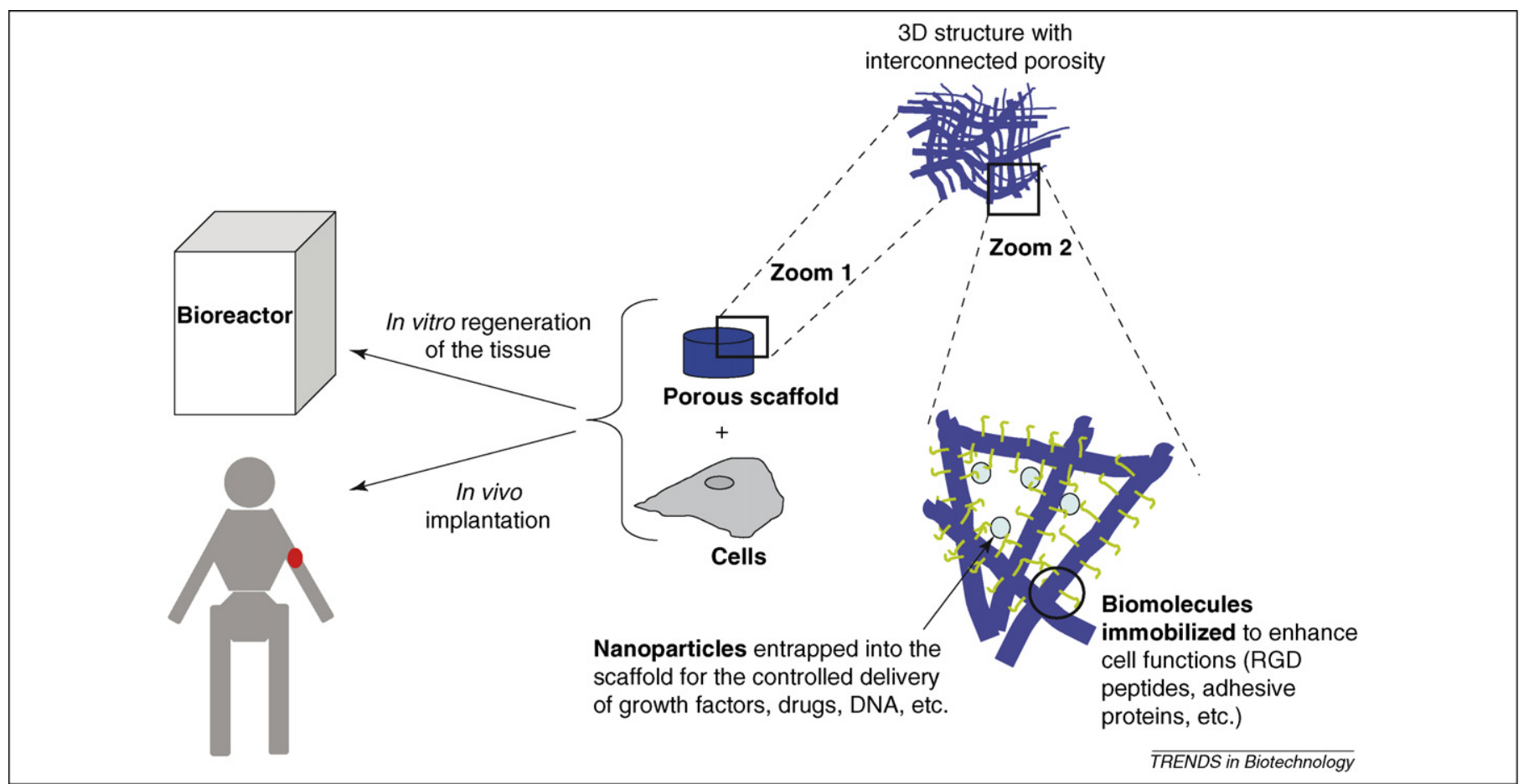

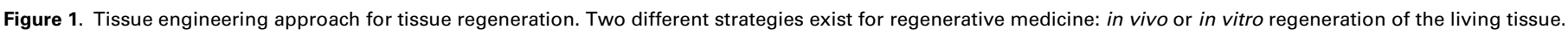
Both methods use a porous scaffold that is loaded with cells. Topographical structure at the nanometre scale can be generated onto the scaffold surface. The scaffold can also be functionalised with different molecules to enhance cell functions. Components, such as drugs, genes, peptide sequences, such as arginine-glycine-aspartic acid (RGD), or proteins, can be entrapped into nanoparticles and released subsequently in a controlled manner to reach a specific target, such as cells, bacteria and viruses.

behaviour greatly [3,4]. In some applications, in which guiding the cell orientation is essential to achieve a functional tissue, such as for tendons, nerves, corneal stroma and intervertebral-disc regeneration, contact guidance of cells by micro- and nano-topographical patterns is a promising perspective $[5,6]$. Chemical cues in the form of various different biomolecules (nanometre scale), such as adhesive protein or growth factors, also influence cell behaviour crucially. For example, fibronectin and laminin are able to enhance the adhesion of different cell types. Therefore, considerable efforts have been made to modify biomaterial surfaces chemically by immobilisation of these biomolecules $[7,8]$.

\section{Nanopattern fabrication}

Because nanotopography seems to have an essential role in guiding cell behaviour in vivo, it is now being used in biomaterials science as a tool for controlling tissue regeneration. A wide variety of techniques have been used to produce nanotopography on biomaterial surfaces. Methods leading to an ordered topography with a regular controlled pattern and methods leading to unordered topography with random orientation and organisation have been developed and are described in Box 1. Polymer demixing, chemical etching and colloidal lithography are some of the most relevant techniques to obtain randomly organised patterns. Conversely, soft-lithography techniques and other methods using different sources of radiation (electrons, ions or photons) to etch the substrate are among the techniques used most commonly to produce regular geometries. Figure 2 shows how specific nanostructured surfaces can be used to control the growth of mesenchymal stem cells (MSCs).
All the techniques of nanostructuration cited here can be combined with self-assembly for functionalisation of the surface (refer to later in the text for the definition and relevance of self-assembly). For example, the creation of a nonfouling surface, which can be useful in ophthalmological and cardiovascular application, can be achieved by the functionalisation of metal surfaces (e.g. Au) with alkanethiol self-assembled molecules, which can bind poly(ethylene-glycol) molecules. Finally, dip-pen lithography is a simple method to create chemical patterns at the nanoscale (Box 1).

\section{Nanocoatings and nanophase materials}

Nanophase materials (i.e. materials with nanometrescaled grains) can be used to produce nanometre features on biomaterial surfaces to guide cell behaviour along a desired biological response $[9,10]$. In bone-regeneration applications, promising results have been obtained with the nanophase materials ceramics and metals, with which increased osteoblast adhesion, proliferation and calcium deposition have been observed compared with conventional materials (i.e. with micrometre-scaled grains) [11].

Nanophase materials can also be deposited on a biomaterial surface to improve its bioactivity and/or biocompatibility. For example, nanocrystalline apatite coatings, produced by sol-gel methods or pulse electrodeposition $[12,13]$, are used commonly to enhance the osteointegration of an orthopaedic device. Other techniques, such as layer-bylayer assembly (LBL) deposition $[14,15]$, lead to nanocoatings (a few nanometres thick) with a controlled structure by sequential adsorption of oppositely charged polyelectrolytes. LBL can be useful for drug-delivery systems [16] and surface functionalisation of biomaterial surfaces [14]. 


\section{Box 1. Methods for topographical and chemical surface modification}

\section{Soft lithography}

Soft lithography is the general term for different techniques based on the use of an elastomeric stamp [which is made of poly(dimethylsiloxane) (PDMS) most commonly] to structure a material surface. This stamp is produced by using a master with a specific pattern (for more details, refer to [48]) and is then used to reproduce the pattern on a substrate.

- Microtransfer moulding: See Figure la for details.

- Replica moulding: The PDMS pattern is used as a mould for casting another polymer, which, after curing, will give a negative replica of the PDMS.

- Micromoulding in capillaries: A PDMS stamp is placed on the substrate to be patterned to form a network of empty channels between the substrate and the stamp. A drop of polymer is placed at one end of the channels and then the channels are filled by capillarity. The PDMS stamp is removed after curing.

- Solvent-assisted micromoulding: A solvent is used to wet the PDMS stamp, which is then placed against the substrate. The substrate is dissolved by the solvent and the resulting fluid is moulded in the PDMS pattern. After evaporation of the solvent and solidification of the substrate, a complementary structure to that of PDMS is obtained.

- Microcontact printing: See Figure lb for details. This is used to transfer molecules from the stamp to a substrate in a controlled geometry.

\section{Colloidal lithography}

This uses a nanocolloidal solution to act as an etch mask. The solution is dispersed as a monolayer on a substrate and self-assembles electrostatically. The surrounding area of the nanocolloid particles (and also of the particles themselves) is etched by reactive ion-beam bombardment, thus creating a pattern on the substrate.

\section{Hot embossing}

A rigid stamp (usually made of silicon) is pressed against a softened polymer film to produce a pattern.

\section{E-beam or ion-beam (EUV) lithography}

This uses particle exposure of a sensitive resist to produce a pattern that can be revealed chemically in a second step or used directly to initiate polymer grafting.

\section{Dip-pen lithography}

This is a direct-write method in which an AFM tip is used in tapping mode to transfer molecules (e.g. proteins, polymers) to a target region of the substrate directly.

\section{Polymer demixing}

This is based on spontaneous phase separation of a polymer mixture under the spin-casting process onto a substrate (silicon, glass). The shape (pit, island, ribbon) and size of the features can be modulated by either changing the polymer ratio or the polymer concentration. $A$ precise control of the height of the features (vertical direction) can be achieved (feature size $>13 \mathrm{~nm}$ ).

(a)

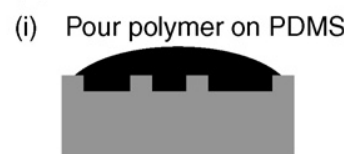

(ii) Remove excess

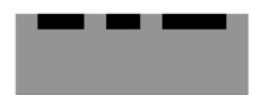

(iii) Put in contact with the substrate

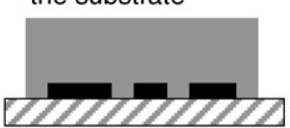

(iv) Wait for curing (UV or heat) and remove PDMS

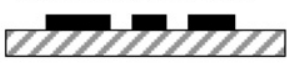

(b)

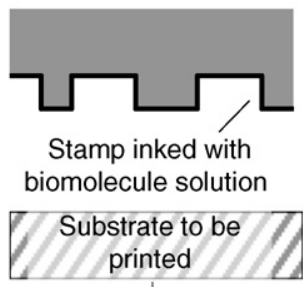

Transfer of biomolecules reproducing the original pattern of the stamp

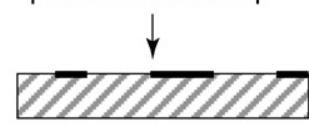

$\overline{\text { TRENDS in Biotechnology }}$

Figure I. (a) Microtransfer moulding. (b) Microcontact printing.

\section{Applications of nanostructured materials}

Numerous in vitro and in vivo studies, using nanostructured materials, have demonstrated the validity of this approach for a variety of cell types. The results of the relevant studies confirmed the potential of nanostructures in regenerating different tissues (such as bone, cartilage, bladder, nerves and vessels) by enhancing the biological performance of the cells (e.g. increased adhesion, proliferation, migration). A summary of some of these applications is given in Table 1.

\section{Materials for nanoparticles}

Nanoparticle research within regenerative medicine has been addressed mainly towards the development of entrapment and delivery systems for genetic material, biomolecules, such as growth and differentiation factors, and bone morphogenetic proteins and also as reinforcing- or bioactivity-enhancement phase for polymeric matrices in $3 \mathrm{D}$ scaffolds for TE.

Controlled delivery of biomolecules is crucial in the support and enhancement of tissue growth in TE applications. Nanotechnology approaches in delivery systems can enhance the success of specific therapeutic agents, such as growth factors and DNA among others, which

are of paramount importance for tissue regeneration [17]. Carriers in the nanoscale enable the intracellular delivery of molecules and the possibility of reaching targets that are inaccessible normally, such as the blood-brain barrier, tight junctions and capillaries, whereas the control over biomolecule dosage and delivery period are increased. The ultimate challenge is to develop artificial nanocarriers that can target cells with efficiency and specificity similar to that of viruses [18].

Examples of nanoparticles for delivery systems include currently microspheres, microcapsules, liposomes, micelles and also dendrimers. The different types of nanoparticles have been developed as solid, hollow or porous. The most common development methods are molecular self-assembly, nanomanipulation, bioaggregation and photochemical patterning $[19,20]$.

Biodegradable polymers are the most commonly used materials in drug delivery. Polylactic acid (PLA), polyglycolic acid (PGA), polyethylene glycol (PEG) and its copolymers have been used widely in combination with hydrogels to attain nanocarriers that exhibit different release properties. Particularly important for the development of nanoparticles for delivery purposes are 'smart' or 'stimuli-responsive' polymers that can undergo 

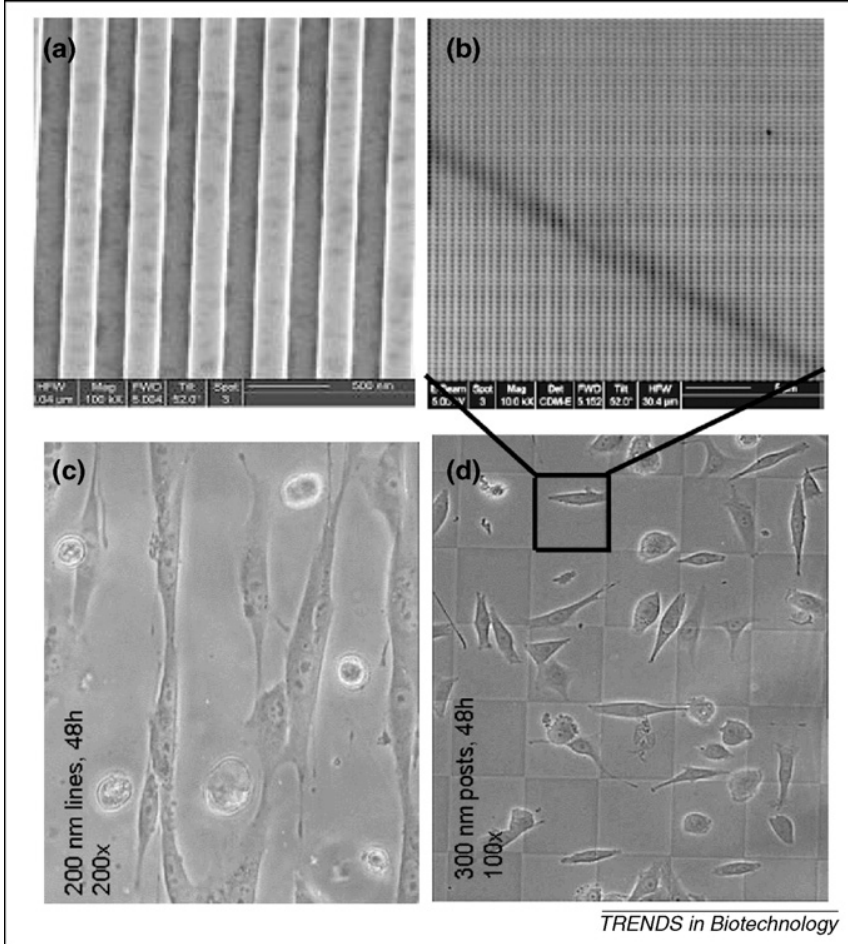

Figure 2. Nanopatterns on polymethylmethacrylate (PMMA) polymer and their influence on mesenchymal stem-cell (MSC) guiding. MSCs from bone marrow were grown on two different nanopatterned surfaces of PMMA for $48 \mathrm{~h}$. (a) A magnified detail of the nanopattern structure, which consisted of $200 \mathrm{~nm}$-wide lines. MSCs grown on this pattern show a directional alignment along the nanostructures (c). (b) Magnification of the square shown in (d) to visualise the pattern of $300 \mathrm{~nm}$-wide posts. Cells growing on this pattern present random distribution and morphology (d).

conformational changes, such as swelling or shrinkage, on variations in temperature, $\mathrm{pH}$ and magnetic field. Table 2 presents several examples of nanoparticles produced with different materials. Their applications in the TE field depend mainly on the material properties.

The most widely studied temperature-sensitive polymer is poly ( $\mathrm{N}$-isopropylacrylamide) (PNIPAAm) [21]. Polyelectrolytes, such as polymethacrylic acid (PMAA) and polyacrylic acid (PAA), that are able to ionise under acidic or alkaline $\mathrm{pH}$ present interesting characteristics for drug carriers in applications in which $\mathrm{pH}$ changes occur, such as in the delivery of genetic material [22]. In the case of genetic-material delivery, nanoscale DNA carriers must overcome enzymatic degradation of endosomes to reach the cell nucleus and trigger specific cascades involved in tissue regeneration. Nanocarriers made of $\mathrm{pH}$-sensitive polymers facilitate endosomal escape and enable the development of new nonviral genetic-material delivery systems with high transfection efficiencies [23].

In addition to biomolecule encapsulation and delivery, solid surface-modified nanoparticles might also be used for regenerative purposes. Although polymers are the most used nanoparticles in the delivery area, the use of ceramics has also been investigated. Hydroxyapatite nanoparticles functionalised with biomolecules could enhance osteoblast adhesion and bone regeneration [9].

Ceramic nanoparticles and nanofibres are also suitable in the elaboration of bio-inspired nanocomposites for bone $\mathrm{TE}$ applications, acting as the reinforcing phase of a polymer matrix and improving scaffold bioactivity [24].

Although the development of nanoparticles seems to have great potential for several biomedical fields, there has been only little progress in the attainment of effective results in current human therapy. However, it must be said that the use of nanoparticles also raises the need of a comprehensive understanding of their secondary effects and cytotoxicity once they enter the body. The large surface area of nanoparticles makes them very reactive in the cellular environment. Their dimensions enable them to penetrate through the lungs, skin or intestinal tract and they might deposit in several organs causing adverse biological reactions [25]. Moreover, the fact that these particles are able to penetrate the cell membrane and reach the cell nucleus might raise some concern about possible unknown risks attributed to the particle nature.

\section{Nanofibre scaffolds}

The first approach of TE was to design and fabricate 3D constructions that could mimic the structure of the tissue that required repair [26]. These scaffolds were made using

Table 1. A summary of nanostructured biomaterials with potential applications in regenerating different tissues

\begin{tabular}{|c|c|c|c|}
\hline Application & Material & Results & Refs \\
\hline \multirow{4}{*}{ Bone } & Titanium coated with nanophase apatite & $\begin{array}{l}\text { Promotion of bone ingrowth and apposition (in vivo) and influence } \\
\text { on human osteoblast behaviour }\end{array}$ & [49] \\
\hline & Tantalum coated with nanophase apatite & $\begin{array}{l}\text { Increased bone formation in comparison with conventional apatite } \\
\text { and uncoated materials (in vivo) }\end{array}$ & [9] \\
\hline & Nanophase $\mathrm{Ti}_{1} \mathrm{Ti}_{6} \mathrm{Al}_{4} \mathrm{~V}$ and CoCrMo alloy & Increased osteoblast adhesion & {$[10,48,50]$} \\
\hline & $\begin{array}{l}\text { Ti mesh coated with nanocrystalline } \\
\text { organoapatite }\end{array}$ & Accelerated migration and better proliferation of osteogenic cells & [52] \\
\hline \multirow[t]{2}{*}{ Cartilage } & Nanostructured PLGA by chemical etching & Better chondrocyte functions than conventional materials & {$[53,54]$} \\
\hline & Nanoporous Ti produced by anodisation & Increased chondrocyte adhesion and migration & [9] \\
\hline Vessels & Nanostructured PLGA by chemical etching & $\begin{array}{l}\text { Increased endothelial and smooth muscle cell densities compared } \\
\text { with untreated PLGA }\end{array}$ & [54] \\
\hline
\end{tabular}


Table 2. Nanoparticles and biomedical applications

\begin{tabular}{|ll|}
\hline Nanoparticles & Applications \\
\hline Titania nanoparticles & Reinforcing phase in composite materials for TE scaffolds [58] \\
Quantum dots & Amplifiers, markers, biological sensors for bioreactors and TE \\
Hydroxyapatite nanoparticles & Reinforcing phase in composite materials for TE, biomolecule carriers [24] \\
Biodegradable polymer nanoparticles & Targeted-delivery systems for TE, immunotherapy [19] \\
Stimuli-sensitive polymeric nanoparticles & Biomolecule carriers, TE [22,59] \\
\hline
\end{tabular}

biodegradable materials and had a porous structure that would ensure host-cell colonisation. Among the possible structures that could replace the natural ECM, the use of nanofibres as scaffolds has several advantages compared with other techniques. Nanofibres show a high surface area and a highly interconnected porous architecture, which facilitate the colonisation of cells in the scaffold and the efficient exchange of nutrients and metabolic waste between the scaffold and its environment. These nanofibres can be made of synthetic, natural or the combination of both types of material. Several relevant methods for the production of nanofibres that are used in $\mathrm{TE}$ applications are summarised here now.

\section{Electrospinning}

Electrospinning is a simple and cost-effective fabrication process that uses an electric field to control the deposition of polymer fibres onto a target substrate. This system can produce fibres with diameters ranging from several microns down to $100 \mathrm{~nm}$ or less. The generated fibres can mimic the structural profile of the proteins found in the native ECM. Different synthetic and natural polymers have been used as materials to create 3D matrices for tissue repair. Some of these polymers have been studied in terms of fibre fabrication, diameter of the fibres and potential applications. The possibility of aligning these fibres is of interest in terms of mimicking the ECM. Different materials have been used to generate such fibres: synthetic biodegradable polymers, such as poly-L-lactic acid (PLLA) [27,28], ع-caprolactone (PCL) [29], PGA [30], and also natural polymers, such as collagen, silk and DNA. The combination of natural and synthetic fibres has been achieved as well. Table 3 summarises some of the materials used and the fibres obtained.

In addition, electrospinning is able to produce both random and aligned networks. This prospect of controlling the orientation of fibres is a prerequirement for biomimicking of natural tissues.

\section{Phase separation}

This process is another method for producing three-dimensional scaffolds of fibres in the submicron range. Current cutting-edge techniques use a thermally induced phaseseparation process to produce a nanofibrous foamed material. However, this method involves several steps, such as raw-material dissolution, gelation, solvent extraction, freezing and drying, which make this process time consuming. The advantages of this method are that it does not require any sophisticated equipment and that the resulting pore sizes and morphology can be controlled by varying the processing parameters [31,32]. The most widely used material for phase-separation foams is biodegradable PLLA, which is sometimes combined with collagen. Yang et al. investigated the potential of PLLA nanofibrous scaffolds prepared by phase separation for nerve TE applications [31]. The resulting scaffold supported cell differentiation and neurite outgrowth. Although this technique is a good approach to mimic the ECM, it has so far failed to control fibre orientation. The distribution and arrangement of the ECM has a crucial role in controlling cell shape, regulating physiological function and defining organ architecture and it is believed that controlling the fibre orientation will increase biomimetics [33].

\section{Self-assembly}

Self-assembly is a process in which molecules and supramolecular aggregates organise and arrange themselves into an ordered structure through weak and noncovalent bonds [34]. The use of self-assembly to generate hierarchical supramolecular structures is a biomimetic strategy to produce synthetic materials that resemble biological ECMs, which are able to interact with cells at the molecular level to control the processes of tissue regeneration effectively. With this technique, nanofibres can be produced using natural or synthetic macromolecules. Several studies report promising results of this strategy. For

Table 3. Nanofibres as scaffolds for TE applications

\begin{tabular}{|c|c|c|c|c|}
\hline Materials & Random/aligned & Average diameter & Potential applications & Refs \\
\hline PLLA & Yes/Yes & $50-350 \mathrm{~nm}$ & Neural TE & {$[28,31]$} \\
\hline Poly(latide-co-glicolide) (PLGA) & Yes/No & $500-800 \mathrm{~nm}$ & Soft tissue & [30] \\
\hline $\mathrm{PCL}$ & Yes/? & $500-900 \mathrm{~nm}$ & Cartilage TE & [29] \\
\hline \multicolumn{5}{|l|}{ Natural polymers } \\
\hline Silk (with HAP/BMP-2) & Yes/No & $?$ & Bone tissue engineering & [62] \\
\hline Chitosan + polyethylene oxide (PEO) & Yes/No & $38-62 \mathrm{~nm}$ & Chondrocites and osteoblasts & [65] \\
\hline Fibrinogen & Yes/No & $320-600 \mathrm{~nm}$ & Cardiac fibroblasts & [66] \\
\hline
\end{tabular}

Abbreviation: HAP/BMP-2, hidroxyapatite/bone morphogenic protein 2. 
example, a peptide amphiphile (chemical compound possessing both hydrophilic and hydrophobic properties) nanofibre network could be mineralised with hydroxyapatite to recreate the nanoscale structure of bone [35]. These amphiphile nanofibres have been designed to mimic the collagen structure-building protein-like structural motifs that incorporate sequences of biological interest [36]. These nanofibres have been also applied to promote rapid and selective differentiation of neural progenitor cells into neurons [37]. Self-assembly was also used successfully to encapsulate chondrocytes within a self-assembled peptidehydrogel scaffold for cartilage repair [38].

\section{Carbon nanotubes}

Although carbon nanotubes (CNs) are not strictly nanofibres, they are another type of nanomaterial that can be used as scaffolds for TE applications. The discovery that carbon could form stable, ordered structures other than graphite and diamond guided Iijima to discover fullerene-related CNs by using an arc-evaporation apparatus [39]. The tubes contained at least two layers (often many more) and ranged in outer diameter from approximately 3 to $30 \mathrm{~nm}$. Since their discovery in 1991, they have been proposed for many $\mathrm{TE}$ applications owing to their exceptional physical properties. These nanomaterials consist of graphite, either in a single layer (singlewalled nanotubes) or in concentric layers (multiwalled carbon nanotubes). The unique properties of $\mathrm{CNs}$, such as the fine electronic, mechanical properties and the high specific surface, might provide a three-dimensional microenvironment to facilitate the use of stem cells in tissue regeneration. [40]. CNs can be prepared by different methods but chemical-vapour deposition (CVD) is used most commonly. This process involves the reaction of a metal catalyst with a hydrocarbon feedstock at high temperatures $\left(>700^{\circ} \mathrm{C}\right)$ [41]. The common use of $\mathrm{Ni}$ as catalyst has raised serious concern about their subsequent use in biomedical applications. In addition, the lack of a scalable production technique for highpurity $\mathrm{CNs}$ has impeded their use in practical applications. However, it has been possible to remove the metal catalyst and to avoid microstructural defects by using CVD at higher temperatures $\left(1800-3000^{\circ} \mathrm{C}\right)$ [40]. Another important issue is that $\mathrm{CNs}$ are not biodegradable and they have to be removed once the tissue has been replaced. CNs have the potential to be used in clinical applications, such as scaffolds for neural implants, because they are highly conductive and are hence an ideal template for transmitting electrical signals to neurons [39]. Moreover, studies on bone engineering have shown that $\mathrm{CN}$ scaffolds are good candidates to support the differentiation of stem cells into mature osteoblasts and the production of bone tissue [40].

Related to health safety, some concerns about using $\mathrm{CNs}$ as scaffolds for TE have been raised. There are reports that demonstrate that nanotubes could be used to separate DNA because DNA strands wrap around CNs. A potential application seems to be separation purposes, although the potential consequences of CNs entering the human body raise a reasonable worry among the scientific community about their safe use in the humans.

\section{Nanodevices}

Recent progress in nanotechnology has led to the development of nanodevices that can act or detect at the nanoscale. This technological advance has been made possible through advances in microfabrication technology, which was developed initially for microelectronic applications. More recently, the fabrication of miomicro-electromechanical systems (BioMEMS) (see [42] for a comprehensive review of BioMEMS) has enabled the application of nanodevices in biomedicine [43]. For the purpose of this review, only BioMEMS with potential uses in regenerative medicine, such as biocapsules, bioreactors, biosensors and laboratory-on-a-chip, will be discussed and evaluated.

Biocapsules are nanodevices in the form of a shell structure that enable the storage and transport of drugs or molecules to be delivered or collected in a controlled manner. Biocapsules can be fabricated to isolate specific molecules. For example, Desai [44] has engineered microfabricated biocapsules with a uniform pore-size distribution that enabled the passage of molecules smaller than $6 \mathrm{~nm}$ but excluded molecules larger than $15 \mathrm{~nm}$. Such a device has a wide range of applications owing to its versatility and simplicity in concept. Thus, the further development of biocapsules can have a tremendous impact in the future of regenerative medicine. They could be used as an accurate and locally sensitive diagnostic tool, for example, when another nanodevice is placed inside the biocapsule, which would be able to analyse entrapped molecules. Such a 'smart' biocapsule could therefore be used as a real-time diagnostic tool and could also be used for the prevention or treatment of a disease when combined with a drug-release system.

Bioreactors are devices that are used in vitro in which regenerative cells and tissues are grown under controlled monitoring and operating conditions (e.g. $\mathrm{pH}$, temperature, pressure, fluidic and mechanical environment, nutrient supply and waste control). These devices integrate several types of BioMEMS into a single device to optimise tissue regeneration and to provide specific conditions for large-scale industrial applications [45]. Biosensors and laboratory-on-a-chip are integrated inside bioreactors to monitor and detect specific cellular processes (Figure 3). Most biosensors available currently are based on microtechnology but advances in nanotechnology foresee the potential for many applications for nanobiosensors. A nanobiosensor is a nanostructure that reacts to the local environment by providing an optical or electrical response. This response is a result of biological, chemical, mechanical or electrical reactions occurring on the sensor. Examples of nanobiosensors are quantum dots, fluorescent nanoparticles, metallic nanoparticles, CNs [41], pH sensors or oxygen sensors that use nanotechnology for locally discrete measurements or a molecule-release sensor (such as release of calcium or potassium) [46]. Energy supply of nanosensors can be provided through the integration of motor proteins that function through ATP [47]. Once nanobiosensors are integrated into bioreactors, they would in theory be able to regulate culture conditions automatically by feedback loops and this would improve tissue regeneration considerably. 


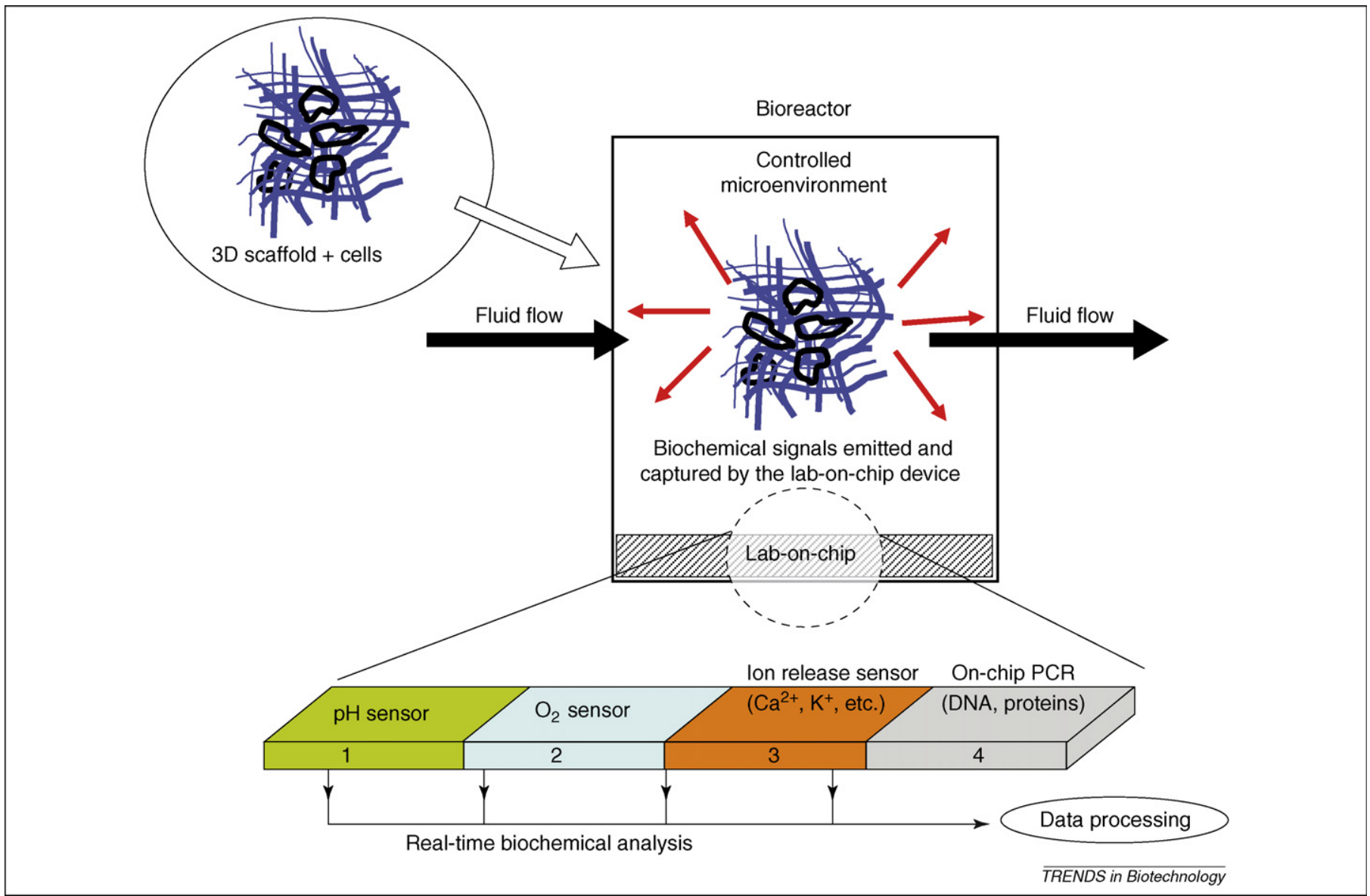

Figure 3. Bioreactors for regenerative medicine. A 3D scaffold with cells is cultured within a bioreactor in which the microenvironment is controlled. The physical and chemical stimuli, which are produced with the application of a controlled fluid flow within the scaffold, induce biochemical signals that are captured by a laboratory-on-achip, in which several nanosensors can be integrated to detect $\mathrm{pH}, \mathrm{O}_{2}$, ions, DNA or proteins. These data can be analysed in real-time to regulate the microenvironments automatically through a feedback-loop mechanism.

\section{Conclusions and future perspectives}

The prospect of replacing damaged tissue with regenerated tissue would impact and change medical science definitely and radically. Today's interest in applying nanotechnology to regenerative medicine is growing owing to its capacity for producing nanostructures that are able to mimic natural tissues as well as nanoparticles for use in delivery systems.

Regenerative medicine aspects that focus on TE have evolved into two main strategies. The first strategy consists of an elegant approach in which stem cells harvested from the patient are expanded and seeded on 3D scaffolds within a bioreactor. The resulting hybrid construct is then implanted into the patient (together with growth factors) as a tissue matrix. However, the need to harvest and expand stem cells poses great efficacy and efficiency problems that define the success of the entire process.

The second strategy relies on the development of intelligent materials that would be able to send signals to the stem cells already present in the diseased or damaged tissue niches that would then trigger the regeneration process. Nanotechnology is a powerful tool for creating these 'smart' materials. This approach is challenging and is still far from being achieved. Among other advantages, it would raise the possibility to have such cell-free materials ready 'off the shelf' and to be able to use them as and when required.
Finally, because nanotechnology is a novel tool, the concern about the safety of nanomaterials used in regenerative medicine for human health has not been addressed in a sufficient and satisfactory manner. Before nanomaterials will be used in human applications, it will be necessary to carry out consistent and comprehensive studies to ensure their innocuousness.

\section{References}

1 Zinger, O. et al. (2005) Differential regulation of osteoblasts by substrate microstructural features. Biomaterials 26, 1837-1847

2 Boyan, B.D. et al. (2002) Osteoblast-mediated mineral deposition in culture is dependent on surface microtopography. Calcif. Tissue Int. 71, $519-529$

3 Hasirci, V. et al. (2006) Nanobiomaterials: a review of the existing science and technology, and new approaches. J. Biomater. Sci. Polym. Ed. 17, 1241-1268

4 Wilkinson, C.D.W. et al. (2002) The use of materials patterned on a nano- and micro-metric scale in cellular engineering. Mater. Sci. Eng. C 19, 263-269

5 Teixeira, A.I. et al. (2006) The effect of environmental factors on the response of human corneal epithelial cells to nanoscale substrate topography. Biomaterials 27, 3945-3954

6 Gomez, N. et al. (2007) Immobilized nerve growth factor and microtopography have distinct effects on polarization versus axon elongation in hippocampal cells in culture. Biomaterials 28, 271-284

7 Massia, S.P. and Stark, J. (2001) Immobilized RGD peptides on surface-grafted dextran promote biospecific cell attachment. $J$. Biomed. Mater. Res. 56, 390-399

8 VandeVondele, S. et al. (2003) RGD-grafted poly-l-lysine-graft(polyethylene glycol) copolymers block non-specific protein 
adsorption while promoting cell adhesion. Biotechnol. Bioeng. 82, 784790

$9 \mathrm{Liu}, \mathrm{H}$. and Webster, T.J. (2007) Nanomedicine for implants: a review of studies and necessary experimental tools. Biomaterials $28,354-369$

10 Webster, T.J. et al. (1999) Osteoblast adhesion on nanophase ceramics. Biomaterials 20, 1221-1227

11 Webster, T.J. and Ejiofor, J.U. (2004) Increased osteoblast adhesion on nanophase metals: $\mathrm{Ti}, \mathrm{Ti}_{6} \mathrm{Al}_{4} \mathrm{~V}$, and CoCrMo. Biomaterials 25, 47314739

12 Saremi, M. and Golshan, B.M. (2007) Microstructural study of nano hydroxyapatite coating obtained by pulse electrodeposition process on Ti-6Al-V-4. Trans. Inst. Metal Finishing 85, 99-102

13 Caruso, R.A. and Antonietti, M. (2001) Sol-Gel nanocoating: an approach to the preparation of structured materials. Chem. Mater. $13,3272-3282$

14 Rudra, J.S. et al. (2006) Antimicrobial polypeptide multilayer nanocoatings. J. Biomater. Sci. Polym. Ed. 17, 1301-1315

$15 \mathrm{Ai}, \mathrm{H}$. et al. (2002) Electrostatic layer-by-layer nanoassembly on biological microtemplates: platelets. Biomacromolecules 3, 560564

16 Kim, B.S. and Choi, J.W. (2007) Polyelectrolyte multilayer microcapsules: self-assembly and toward biomedical applications. Biotechnol. Bioprocess Eng. 12, 323-332

17 Reddy, S.T. et al. (2006) In vivo targeting of dendritic cells in lymph nodes with poly(propylene sulfide) nanoparticles. J. Control. Release $112,26-34$

18 Mastrobattista, E. et al. (2006) Artificial viruses: a nanotechnological approach to gene delivery. Nat. Rev. Drug Discov. 5, 115-122

19 Alleman, E. et al. (1993) Drug-loaded nanoparticles-preparation methods and drug targeting issues. Eur. J. Pharm. Biopharm. 39, 173-191

20 Cade, D. et al. (2004) Tailoring of bioresorbable polymers for elaboration of sugar-functionalized nanoparticles. Biomacromolecules $5,922-927$

21 Gil, E.S. and Hudson, S.M. (2004) Stimuli responsive polymers and their conjugates. Prog. Polym. Sci. 29, 1173-1222

22 Murthy, N. et al. (2003) Design and synthesis of $\mathrm{pH}$ responsive polymeric carriers that target uptake and enhance the intracellular delivery of oligonucleotides. J. Control. Release 89, 365-374

23 Langer, R. and Tirrel, D.A. (2004) Designing materials for biology and medicine. Nature 428, 487-492

24 Sarvestani, A. et al. (2006) Effect of osteonectin-derived peptide on the viscoelasticity of hydrogel/apatite nanocomposite scaffolds. Biopolymers 85, 370-378

25 Medina, C. et al. (2007) Nanoparticles: pharmacological and toxicological significance. Br. J. Pharmacol. 150, 552-558

26 Lanza, R.P. et al. (2000) Principles of Tissue Engineering. (2nd edn), Academic Press

27 Bognitzki, M. et al. (2002) Nanostructured fibers via electrospinning. Adv. Mater. 13, 70-72

28 Yang, F. et al. (2005) Electrospinning of nano/micro scale poly(L-lactic acid) aligned fibers and their potential in neural tissue engineering. Biomaterials 26, 2603-2610

$29 \mathrm{Li}$, W-J. et al. (2005) A three-dimensional nanofibrous scaffold for cartilage tissue engineering using human mesenchymal stem cells. Biomaterials 26, 599-609

$30 \mathrm{Li}$, W-J. et al. (2002) Electrospun nanofibrous structure: a novel scaffold for tissue engineering. J. Biomed. Mater. Res. 60, 613621

31 Yang, F. et al. (2004) Fabrication of nano-structured porous PLLA scaffold intended for nerve tissue engineering. Biomaterials 25, 18911900

32 Wei, G. and Ma, P.X. (2004) Structure and properties of nanohydroxiapatite/polymer composite scaffolds for bone tissue engineering. Biomaterials 25, 4749-4757

33 Matthews, J.A. et al. (2002) Electrospinning of collagen nanofibers. Biomacromolecules 3, 232-238

34 Murugan, R. and Ramakrishna, S. (2007) Design strategies of tissue engineering scaffolds with controlled fiber orientation. Tissue Eng. 13, $1845-1866$

35 Hartgerink, J.D. et al. (2001) Self assembly and mineralization of peptide-amphibile nanofibers. Science 294, 1684-1688
36 Fields, G. et al. (1998) Proteinlike molecular architecture: biomaterial applications for inducing cellular receptor binding and signal transduction. Peptide Sci. 47, 143-151

37 Silva, G.A. et al. (2004) Selective differentiation of neural progenitor cells by high-epitope density nanofibers. Science 303, 1352-1355

38 Kisiday, J. et al. (2002) Self-assembling peptide hydrogel fosters chondrocyte extracellular matrix production and cell division: implications for cartilage tissue repair. Proc. Natl. Acad. Sci. U. S. A. $99,9996-10001$

39 Iijima, S. (1991) Helical microtubules of graphitic carbon. Nature 354, $56-58$

40 Andrews, R. et al. (2001) Purification and structural annealing of multiwalled carbon nanotubes at graphitization temperatures. Carbon 39, 1681-1687

41 Harrison, B.S. and Atala, A. (2007) Carbon nanotube applications for tissue engineering. Biomaterials 28, 344-353

42 Bashir, R. (2004) BioMEMS: state-of-the-art in detection, opportunities and prospects. Adv. Drug Deliv. Rev. 56, 1565-1586

43 Khademhosseini, A. et al. (2006) Interplay of biomaterials and microscale technologies for advancing biomedical applications. J. Biomater. Sci. Polym. Ed. 17, 1221-1240

44 Desai, T.A. (2000) Micro- and nanoscale structures for tissue engineering constructs. Med. Eng. Phys. 22, 595-606

45 Martin, I. et al. (2004) The role of bioreactors in tissue engineering. Trends Biotechnol. 22, 80-86

46 Bishnoi, S.W. et al. (2006) All-optical nanoscale pH meter. Nano Lett. 6 , 1687-1692

$47 \mathrm{Liu}, \mathrm{H}$. et al. (2002) Control of a biomolecular motor-powered nanodevice with an engineered chemical switch. Nat. Mater. 1, 173177

48 Xia, Y. and Whitesides, G.M. (1998) Soft lithography. Annu. Rev. Mater. Sci. 28, 153-184

$49 \mathrm{Li}, \mathrm{P}$. (2003) Biomimetic nano-apatite coating capable of promoting bone ingrowth. J. Biomed. Mater. Res. A 66, 79-85

50 Faghihi, S. et al. (2007) Cellular and molecular interactions between MC3T3-E1 pre-osteoblasts and nanostructured titanium produced by high-pressure torsion. Biomaterials 28, 3887-3895

51 Palin, E. et al. (2005) Mimicking the nanofeatures of bone increases bone-forming cell adhesion and proliferation. Nanotechnology 16 , 1828-1835

52 Spoerke, E.D. and Stupp, S.I. (2003) Colonization of organoapatitetitanium mesh by preosteoblastic cells. J. Biomed. Mater. Res. A 67, 960-969

53 Kay, S. et al. (2002) Nanostructured polymer/nanophase ceramic composites enhance osteoblast and chondrocyte adhesion. Tissue Eng. 8, 753-761

54 Miller, D.C. et al. (2004) Endothelial and vascular smooth muscle cell function on poly(lactic-co-glycolic acid) with nano-structured surface features. Biomaterials 25, 53-61

55 Thapa, A. et al. (2003) Nano-structured polymers enhance bladder smooth muscle cell function. Biomaterials 24, 2915-2926

56 Thapa, A. et al. (2003) Polymers with nano-dimensional surface features enhance bladder smooth muscle cell adhesion. J. Biomed. Mater. Res. A 67, 1374-1383

57 Yim, E.K.F. et al. (2007) Synthetic nanostructures inducing differentiation of human mesenchymal stem cells into neuronal lineage. Exp. Cell Res. 313, 1820-1829

58 Fan, X. et al. (2006) Surface-initiated polymerization from $\mathrm{TiO}_{2}$ nanoparticle surfaces through a biomimetic initiator: A new route towards polymer-matrix nanocomposites. Compos. Sci. Technol. 66, 1198-1204

59 Lo, C.L. et al. (2005) Preparation and characterization of intelligent core-shell nanoparticles based on poly(D,L-lactide)-g-poly $(\mathrm{N}$ isopropylacrylamide-co-methacrylic acid). J. Control. Release 104, 477-488

60 Mo, X.M. et al. (2004) LLA_CL) nanofiber: a biomimetic extracellular matrix for smooth muscle cell and endothelial cell proliferation. Biomaterials 25, 1883-1890

$61 \mathrm{Xu}$, C.Y. et al. (2004) Aligned biodegradable nanofibrous structure: a potential scaffold for blood vessel engineering. Biomaterials 25, 877886

$62 \mathrm{Li}$, C. et al. (2005) Silk apatite composites from electrospun fibers. J. Mater. Res. 20, 3374-3384 
63 Zhong, S. et al. (2006) An aligned nanofibrous collagen scaffold by electrospinning and its effects on in vitro fibroblast culture. J. Biomed. Mater. Res. A 79, 456-463

64 Ohkawa, K. et al. (2004) Electrospinning of chitosan. Macromol. Rapid Commun. 25, 1600-1605
65 Bhattarai, S.R. et al. (2004) Novel biodegradable electrospun membrane: scaffold for tissue engineering. Biomaterials 25, 2595-2602

66 McManus, M.C. et al. (2007) Electrospun fibrinogen: feasibility as a tissue engineering scaffold in a rat cell culture model. J. Biomed. Mater. Res. A 81, 299-309

\section{Have you contributed to an Elsevier publication? Did you know that you are entitled to a $30 \%$ discount on books?}

A 30\% discount is available to all Elsevier book and journal contributors when ordering books or stand-alone CD-ROMs directly from us.

To take advantage of your discount:

1. Choose your book(s) from www.elsevier.com or www.books.elsevier.com

\section{Place your order}

Americas:

Phone: +1800 7824927 for US customers

Phone: +1 8004603110 for Canada, South and Central America customers

Fax: +1 3144534898

author.contributor@elsevier.com

All other countries:

Phone: $+44(0) 1865474010$

Fax: $+44(0) 1865474011$

directorders@elsevier.com

You'll need to provide the name of the Elsevier book or journal to which you have contributed. Shipping is free on prepaid orders within the US.

If you are faxing your order, please enclose a copy of this page.

\section{Make your payment}

This discount is only available on prepaid orders. Please note that this offer does not apply to multi-volume reference works or Elsevier Health Sciences products.

For more information, visit www.books.elsevier.com 